\title{
Nigrostriatal Degeneration in Parkinson Disease: Evaluation by Diffusion Tensor Tract-Specific Analysis
}

\author{
Alina Abulaiti', Koji Kamagata1, Yumiko Motoi ${ }^{2}$, Masaaki Hori' ${ }^{1}$, Nobutaka Hattori², \\ Shigeki Aoki ${ }^{1}$ \\ ${ }^{1}$ Department of Radiology, Juntendo University Graduate School of Medicine, Tokyo, Japan \\ ${ }^{2}$ Department of Neurology, Juntendo University Graduate School of Medicine, Tokyo, Japan \\ Email:kkamagat@juntendo.ac.jp
}

Received 5 October 2015; accepted 1 December 2015; published 4 December 2015

Copyright (C) 2015 by authors and Scientific Research Publishing Inc.

This work is licensed under the Creative Commons Attribution International License (CC BY).

http://creativecommons.org/licenses/by/4.0/

(c) (i) Open Access

\begin{abstract}
Diffusion tensor tractography was used to evaluate whether diffusion metrics in the nigrostriatal pathway could diagnose Parkinson disease. Diffusion tensor imaging was performed on 30 patients with Parkinson disease and 32 healthy controls by using a 3.0 Tesla magnetic resonance imaging system. Diffusion tensor tractography was used for both groups to visualize the nigrostriatal and corticospinal tracts. The fractional anisotropy (FA) and mean diffusivity (MD) of the tracts were evaluated. Receiver operating characteristic (ROC) analysis was used to determine whether diffusion metrics of the nigrostriatal pathway could be used to diagnose Parkinson disease. Mean FA values $( \pm S D)$ of the nigrostriatal tract in Parkinson disease patients $(0.41 \pm 0.025)$ were significantly lower than those in the control group $(0.43 \pm 0.022 ; p=0.00068)$ and showed a sensitivity of $66.7 \%$ and specificity of $60 \%$. There were no significant differences in the MD values of the nigrostriatal tract or the FA and MD values of the corticospinal tract between Parkinson disease patients and the control group. FA values of the nigrostriatal pathway in Parkinson disease patients were significantly lower than those in normal, healthy individuals. Reduced FA was generally thought to reflect neuronal loss, gliosis, or demyelination of nerve fibers. This result might provide a useful measure for diagnosing Parkinson disease and evaluating patients' clinical condition.
\end{abstract}

\section{Keywords}

Nigrostriatal Pathway, Diffusion MRI, Neurodegenerative Disorders, Parkinson Disease 


\section{Introduction}

Parkinson disease is the second most common neurodegenerative disorder after Alzheimer disease [1]. The brain abnormalities of Parkinson disease are difficult to detect by using conventional magnetic resonance (MR) imaging [2]. Diffusion tensor imaging (DTI) [3] [4] and other advanced diffusion techniques [5] have been reported to be useful for detecting the subtle abnormalities of the brains of Parkinson disease patients [6] [7]. The main pathological characteristic of Parkinson disease is the selective loss of nigrostriatal dopaminergic projections [8]. Although several authors have reported abnormal diffusion metrics of the related regions [9], only one previous report has shown the use of diffusion tensor tractography to evaluate the nigrostriatal pathway [4] [10]. The purpose of the present study is to delineate and quantify the nerve fibers that connect the substantia nigra and striatum (including the nigrostriatal dopaminergic projections) by using diffusion tensor tractography.

\section{Materials and Methods}

\subsection{Subjects}

We enrolled 30 patients with Parkinson disease (mean age, 69.7 years) and 32 healthy age- and sex-matched controls (mean age, 68.0 years). The demographic characteristics of the subjects are shown in Table 1 . This study was approved by our institution's review board, and informed consent was obtained from all participants before evaluation. In all patients with Parkinson disease, the disease had been diagnosed by neurologists and fulfilled the UK Parkinson’s Disease Society Brain Bank criteria.

\subsection{MR Imaging}

All participants were examined by using a 3.0 Tesla MR imaging system (Achieva, Philips Healthcare, Best, The Netherlands) and an 8-channel array receiving head coil. Conventional structural MR sequences of T1-weighted, T2-weighted, FLAIR, and 3D T1-weighted images (MP-RAGE, $1 \mathrm{~mm}$ cubic voxel) were acquired. Diffusion tensor imaging was performed by using the spin-echo echo-planar technique (TR/TE, 5443/70 ms; matrix size, $128 \times 128$; FOV, $224 \times 224 \mathrm{~mm}^{2}$; section thickness, $3 \mathrm{~mm}$ with no gap). Images were obtained along 32 different directions with a b factor of $1000 \mathrm{~s} / \mathrm{mm}^{2}$ and no diffusion encoding (b: $0 \mathrm{~s} / \mathrm{mm}^{2}$ ). A total of 50 axial section images covering the whole cerebrum were obtained. The scanning time was 7 minutes 17 seconds. Diffusion tensor data were transferred to an off-line workstation. Maps of fractional anisotropy (FA) and mean diffusivity (MD) were computed by using the dTVII

(http://www.medimg.info.hiroshima-cu.ac.jp/dTV.II.15g/index.html) and VOLUME-ONE software [11]. For diffusion tensor tractography of the nigrostriatal tract, the seed and target were set in the substantia nigra and corpus striatum, respectively (Figure 1). In determining the FA threshold for the nigrostriatal tract, we examined 10 subjects chosen randomly from among the normal controls of the present study. We adopted an FA threshold of 0.10 , with which the nigrostriatal tract was most appropriately extracted by visual assessment. After checking the visualized tract, FA and MD between the seed and the target were recorded. For diffusion tensor tractography of the corticospinal tract, the seed and target were set in the cerebral peduncle and precentral gyrus, respectively, as described elsewhere [12]. In corticospinal tract, The FA threshold for tracking was set at 0.18 in accordance with a previous report by Yasmin et al. [13] The FA and MD at the level of the basal ganglia were evaluated.

\subsection{Statistical Analysis}

All statistical analyses were performed with the Statistical Package for the Social Sciences for Windows, Release 20.0 (SPSS, Chicago, Illinois). Statistical analysis of demographic and clinical data was conducted with Student's $t$-test for continuous variables and the $\chi^{2}$ test for categorical data. The criterion of statistical significance

Table 1. Demographic characteristics of the patients enrolled in the present study.

\begin{tabular}{cccc}
\hline & HC $(n=32)$ & PD $(n=30)$ & $p$ value \\
\hline Sex, male: female & $19: 13$ & $13: 17$ & NS \\
Age in years, mean (SD) & $68.1(10.2)$ & $69.7(6.7)$ & NS \\
\hline
\end{tabular}



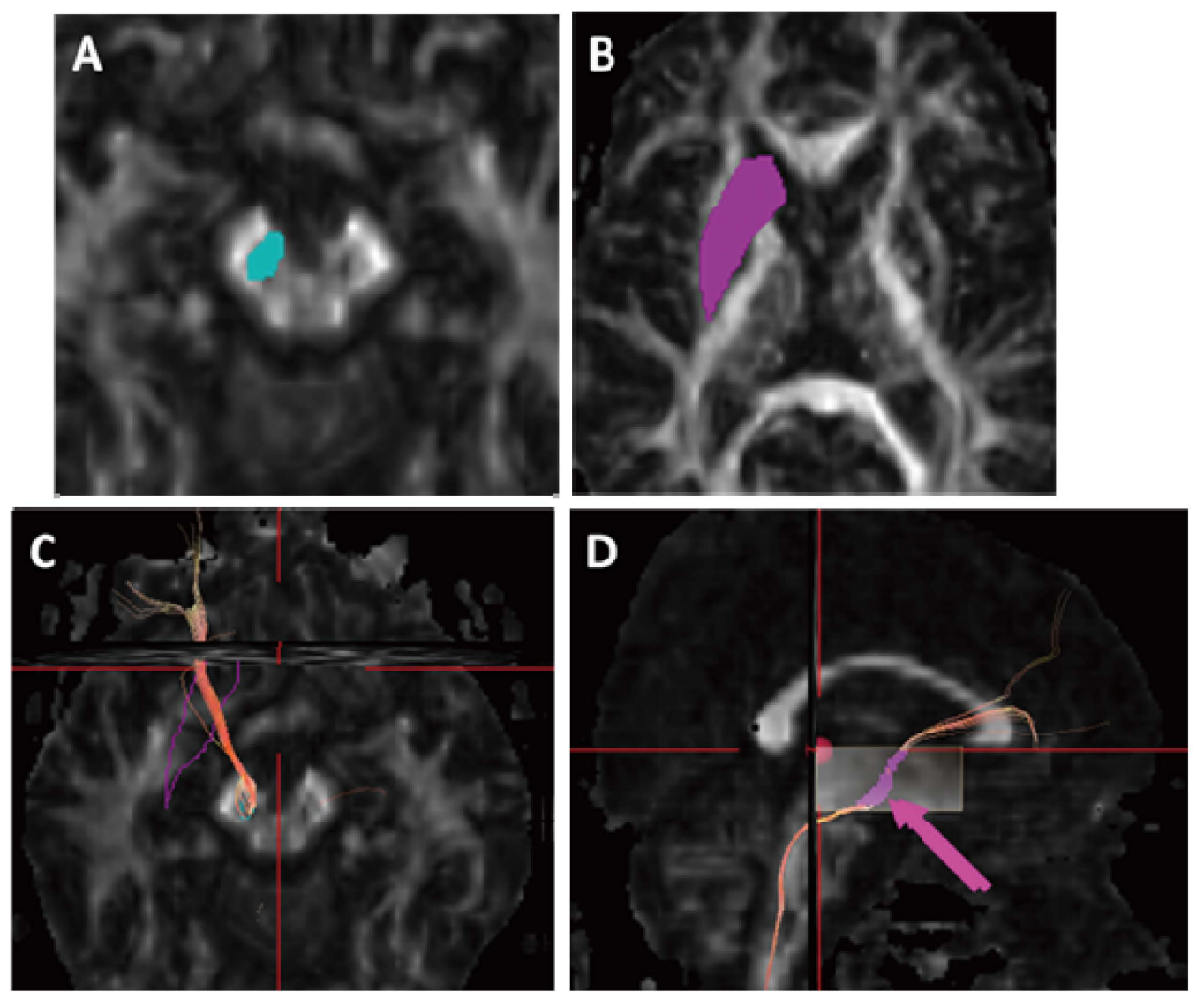

Figure 1. Diffusion tensor tractography images of the right nigrostriatal projection, and voxelization alongwith diffusion tensor tractography images of the nigrostriatal projection. (A) The seed region of interest (ROI), including the entirety of the substantia nigra (light blue area), was placed manually on an axial section of the fractional anisotropy (FA) maps; (B) The target ROI, including the entirety of the corpus striatum (purple area), was placed manually on a axial section of the FA maps; (C) Tractographic image of the right nigrostriatal projection was generated from the seed ROI to the target ROI; (D) In this study, the nigrostriatal projection was defined between the seed ROI and the target ROI. Voxelization was performed along the right nigrostriatal projection between the seed ROI and the target ROI (blue voxels), and FA values in coregistered voxels were calculated.

was $p<0.05$. The unpaired $t$-test was used to compare the measured values for FA and MD between the groups. The Bonferroni correction was used for multiple comparisons $(n=4$ : [nigrostriatal tract, corticospinal tract] $\times$ [FA, MD], setting the level of significance at $p<0.05 / 4=0.0125$ ). Receiver operating characteristic (ROC) analysis was used to determine whether diffusion metrics of the nigrostriatal pathway can be used to diagnose Parkinson disease.

\section{Results}

Age and sex distributions did not differ between patients with Parkinson disease and healthy controls (Table 1). The mean FA values ( \pm standard deviation [SD]) of the nigrostriatal tract in patients with Parkinson disease $(0.408 \pm 0.025)$ were significantly lower than those in the control group $(0.430 \pm 0.022 ; p=0.00068)$ (Table 2).

The mean MD values were slightly higher in patients with Parkinson disease $\left(0.771 \pm 0.052 \mathrm{~mm}^{2} / \mathrm{s}\right)$ compared with those in the control group $\left(0.741 \pm 0.073 \mathrm{~mm}^{2} / \mathrm{s}\right)$, but the difference was not significant (Table 2).

There were no significant differences between the mean FA and MD values of the corticospinal tracts in patients with Parkinson disease $\left(0.722 \pm 0.028,0.745 \pm 0.027 \mathrm{~mm}^{2} / \mathrm{s}\right)$ and those in the control group $(0.709 \pm$ $0.029,0.730 \pm 0.031 \mathrm{~mm}^{2} / \mathrm{s}$ ) (Table 2). The ROC analysis, using a cutoff value of 0.418 for the FA value of the nigrostriatal pathway, showed a sensitivity of $66.7 \%$ and specificity of $60 \%$ (Figure 2). 
Table 2. Comparison of mean diffusivity (MD) and fractional anisotropy (FA) in patients and control subjects.

\begin{tabular}{cccc}
\hline & HC & PD & p value \\
\hline Nigrostriatal tract & & & $0.00068^{\mathrm{b}}$ \\
FA & $0.430 \pm 0.022^{\mathrm{a}}$ & $0.408 \pm 0.025$ & 0.070 \\
MD $^{\mathrm{c}}$ & $0.741 \pm 0.073$ & $0.771 \pm 0.052$ & 0.082 \\
Corticospinal tract & & & $0.722 \pm 0.028$ \\
FA & $0.709 \pm 0.029$ & $0.745 \pm 0.027$ & 0.043 \\
\hline
\end{tabular}

Note: HC, healthy controls; PD, Parkinson disease. ${ }^{\mathrm{a}}$ Values are mean $\pm \mathrm{SD} .{ }^{\mathrm{b}}$ Result statistically significant $(p<0.0125) .{ }^{\mathrm{c}}$ Mean diffusivity values expressed as $10^{-3} \mathrm{~mm}^{2} / \mathrm{s}$.

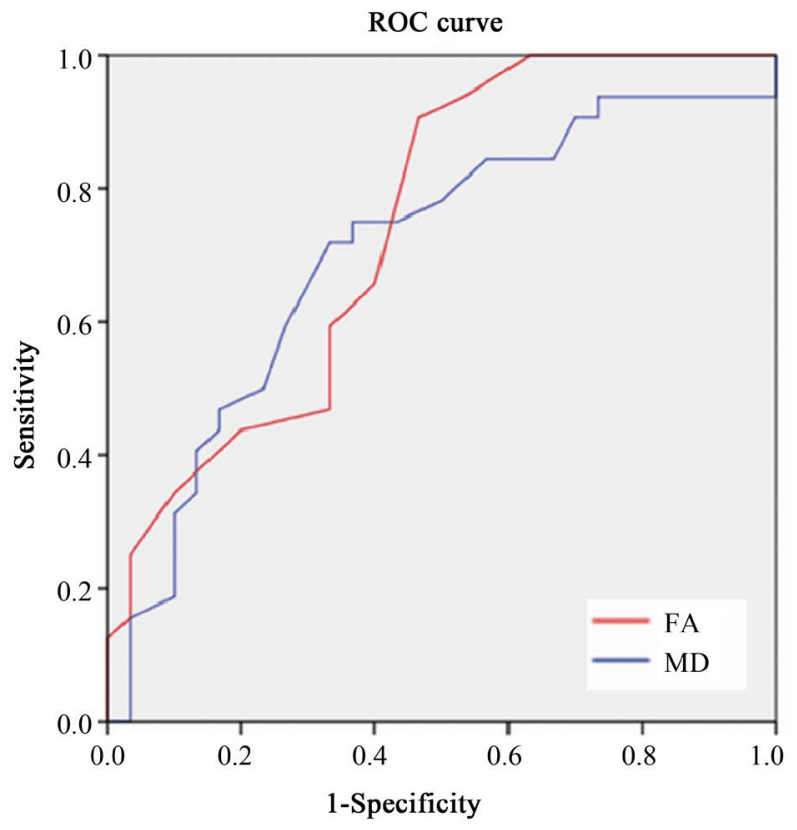

Figure 2. Receiver operating characteristic plots for fractional anisotropy (FA) and mean diffusivity (MD) at the nigrostriatal projection. FA had the greatest sensitivity and specificity.

\section{Discussion}

The nigrostriatal pathway, an important dopaminergic pathway that connects the substantia nigra and the striatum, is the main focus of Parkinson pathology [8] [14]. Degeneration of this pathway has been recently assessed by diffusion tensor [4] [10] and other diffusion MR techniques that use ROI analyses [5]. In the present study, we directly assessed the nigrostriatal pathway using diffusion tensor tractography, which can be used to noninvasively visualize white matter tracts and is therefore a powerful tool to visualize connectivity of the brain in vivo [15] [16]. Tract-specific analysis (TSA), or tract of interest analysis, is a type of ROI/VOI (volume of interest) analysis that measures diffusion metrics within the visualized tract. TSA can be considered a semi-automatic ROI setting analysis because two ROIs are located manually and VOIs between them are automatically selected by the diffusion tensor tractography algorithm [17]. It is also a highly reliable technique because the location of the VOIs can be validated visually by means of 3D diffusion tensor tractography. The inter-observer correlation for TSA is high [18].

A well-known limitation of diffusion tensor tractography is the crossing or kissing of fibers within a voxel. The visualized tract can be considered an actual tract under the limited conditions that no significant fiber crossing or kissing occurs along the pathway. Although we suspect that the region defined here as being involved in diffusion tensor tractography of the nigrostriatal tract actually includes the nigrostriatal dopaminergic 
neurons, it may also include projection fibers from the corticospinal tract and other regions. However a previous report that the corticospinal tract and other main projection fibers are not significantly altered in Parkinson disease [19], we confirmed that the diffusion metrics of the corticospinal tracts of Parkinson disease patients do not differ from those of control subjects. Therefore we surmise that the FA decrease of the nigrostriatal diffusion tensor tractography in patients with PD mainly reflects the influence of the dopaminergic nigrostriatal fibers.

In this study, reduced FA is generally thought to reflect neuronal loss, gliosis, or demyelination of nerve fibers. Histopathological studies have shown that nigrostriatal dopaminergic projections have already decreased in number by half at the time of disease onset [20].

Our findings of reduced FA of the nigrostriatal diffusion tensor tractography might reflect the loss of neurons, including the nigrostriatal dopaminergic projection neurons [14]. Although previous DTI studies have set the substantia nigra or corpus striatum as the ROI, only one study has investigated the projections that connect the substantia nigra and corpus striatum by using diffusion tensor tractography [10]. As in the present study, Zhang et al., by TSA that utilized deterministic tractography, have reported a decline in the FA for diffusion tensor tractography of the nigrostriatal tract [10]. Here we confirmed similar results and further demonstrated the actual diagnostic ability of this technique by ROC analysis.

By using a VOI approach for tractography, the location of a visual measurement can be easily checked. This approach may be useful for the early diagnosis of Parkinson disease.

The limitations of this study are its small sample size, relatively large voxel size for DTI metrics, and manual setting of seed and target ROIs. The limitations of voxel size and motion probing gradient directions are difficult to solve in the clinical setting. The multiband echo-planar technique, which shortens the scan time and increases the spatial and directional resolution of DTI, is garnering increased attention. We are planning a new protocol using this technique.

\section{Conclusion}

The FA of the nigrostriatal pathway was significantly lower in Parkinson disease patients than in normal control subjects. This difference might reflect the degeneration and loss of nigrostriatal dopaminergic projections and might, therefore provide a useful measure for diagnosing Parkinson disease.

\section{Acknowledgements}

We thank Nozomi Hamasaki and Syuji Sato, MR imaging technologists, for their skillful acquisition of data; Akira Nishikori, Tomomi Okamura, and Fumitaka Kumagai for their research assistance; and Yuriko Suzuki and Masaru Takashima of Philips Healthcare for their technical assistance. We also thank Narisumi Cho for administrative assistance. This work was supported by a research grant from Hitachi, Ltd.; the program for Brain Mapping by Integrated Neurotechnologies for Disease Studies (Brain/MINDS) from Japan Agency for Medical Research and development, AMED; Technology of Japan and by MEXT/JSPS KAKENHI Grant Number 26893266.

\section{References}

[1] de Lau, L.M. and Breteler, M.M. (2006) Epidemiology of Parkinson’s Disease. The Lancet Neurology, 5, 525-535. http://dx.doi.org/10.1016/S1474-4422(06)70471-9

[2] Peran, P., Cherubini, A., Assogna, F., Piras, F., Quattrocchi, C., et al. (2010) Magnetic Resonance Imaging Markers of Parkinson's Disease Nigrostriatal Signature. Brain, 133, 3423-3433. http://dx.doi.org/10.1093/brain/awq212

[3] Blain, C.R., Barker, G.J., Jarosz, J.M., et al. (2006) Measuring Brain Stem and Cerebellar Damage in Parkinsonian Syndromes Using Diffusion Tensor MRI. Neurology, 67, 2199-2205.

[4] Yoshikawa, K., Nakata, Y., Yamada, K. and Nakagawa, M. (2004) Early Pathological Changes in the Parkinsonian Brain Demonstrated by Diffusion Tensor MRI. Journal of Neurology, Neurosurgery \& Psychiatry, 75, 481-484. http://dx.doi.org/10.1136/jnnp.2003.021873

[5] Wang, J.J., Lin, W.Y., Lu, C.S., et al. (2011) Parkinson Disease: Diagnostic Utility of Diffusion Kurtosis Imaging. Radiology, 261, 210-217. http://dx.doi.org/10.1148/radiol.11102277

[6] Gattellaro, G., Minati, L., Grisoli, M., et al. (2009) White Matter Involvement in Idiopathic Parkinson Disease: A Diffusion Tensor Imaging Study. American Journal of Neuroradiology, 30, 1222-1226. http://dx.doi.org/10.3174/ajnr.A1556 
[7] Vaillancourt, D.E., Spraker, M.B., Prodoehl, J., et al. (2009) High-Resolution Diffusion Tensor Imaging in the Substantia Nigra of de Novo Parkinson Disease. Neurology, 72, 1378-1384. http://dx.doi.org/10.1212/01.wnl.0000340982.01727.6e

[8] O’Reilly, S., Loncin, M. and Cooksey, B. (1965) Dopamine and Basal Ganglia Disorders. Neurology, 15, 980-984. http://dx.doi.org/10.1212/WNL.15.11.980

[9] Chan, L.L., Rumpel, H., Yap, K., et al. (2007) Case Control Study of Diffusion Tensor Imaging in Parkinson’s Disease. Journal of Neurology, Neurosurgery \& Psychiatry, 78, 1383-1386. http://dx.doi.org/10.1136/jnnp.2007.121525

[10] Zhang, Y., Wu, I.W., Buckley, S., et al. (2015) Diffusion Tensor Imaging of the Nigrostriatal Fibers in Parkinson’s Disease. Movement Disorders, 30, 1229-1236. http://dx.doi.org/10.1002/mds.26251

[11] Masutani, Y., Aoki, S., Abe, O., Hayashi, N. and Otomo, K. (2003) MR Diffusion Tensor Imaging: Recent Advance and New Techniques for Diffusion Tensor Visualization. European Journal of Radiology, 46, 53-66. http://dx.doi.org/10.1016/S0720-048X(02)00328-5

[12] Yasmin, H., Aoki, S., Abe, O., et al. (2009) Tract-Specific Analysis of White Matter Pathways in Healthy Subjects: A Pilot Study Using Diffusion Tensor MRI. Neuroradiology, 51, 831-840. http://dx.doi.org/10.1007/s00234-009-0580-1

[13] Yasmin, H., Nakata, Y., Aoki, S., et al. (2008) Diffusion Abnormalities of the Uncinate Fasciculus in Alzheimer's Disease: Diffusion Tensor Tract-Specific Analysis Using a New Method to Measure the Core of the Tract. Neuroradiology, 50, 293-299. http://dx.doi.org/10.1007/s00234-007-0353-7

[14] Boska, M.D., Hasan, K.M., Kibuule, D., et al. (2007) Quantitative Diffusion Tensor Imaging Detects Dopaminergic Neuronal Degeneration in a Murine Model of Parkinson's Disease. Neurobiology of Disease, 26, 590-596. http://dx.doi.org/10.1016/j.nbd.2007.02.010

[15] Mori, S. and van Zijl, P.C. (2002) Fiber Tracking: Principles and Strategies-A Technical Review. NMR in Biomed, 15, 468-480. http://dx.doi.org/10.1002/nbm.781

[16] Kunimatsu, A., Aoki, S., Masutani, Y., Abe, O., Mori, H. and Ohtomo, K. (2003) Three-Dimensional White Matter Tractography by Diffusion Tensor Imaging in Ischaemic Stroke Involving the Corticospinal Tract. Neuroradiology, 45, 532-535. http://dx.doi.org/10.1007/s00234-003-0974-4

[17] Aoki, S., Iwata, N.K., Masutani, Y., et al. (2005) Quantitative Evaluation of the Pyramidal Tract Segmented by Diffusion Tensor Tractography: Feasibility Study in Patients with Amyotrophic Lateral Sclerosis. Radiation Medicine, 23, 195-199.

[18] Kamagata, K., Motoi, Y., Abe, O., et al. (2012) White Matter Alteration of the Cingulum in Parkinson Disease with and without Dementia: Evaluation by Diffusion Tensor Tract-Specific Analysis. American Journal of Neuroradiology, 33, 890-895. http://dx.doi.org/10.3174/ajnr.A2860vv

[19] Abe, O., Aoki, S., Hayashi, N., et al. (2002) Normal Aging in the Central Nervous System: Quantitative MR Diffusion-Tensor Analysis. Neurobiology of Aging, 23, 433-441. http://dx.doi.org/10.1016/S0197-4580(01)00318-9

[20] Braak, H., Del Tredici, K., Rub, U., de Vos, R.A., Jansen Steur, E.N. and Braak, E. (2003) Staging of Brain Pathology Related to Sporadic Parkinson's Disease. Neurobiology of Aging, 24, 197-211. http://dx.doi.org/10.1016/S0197-4580(02)00065-9 